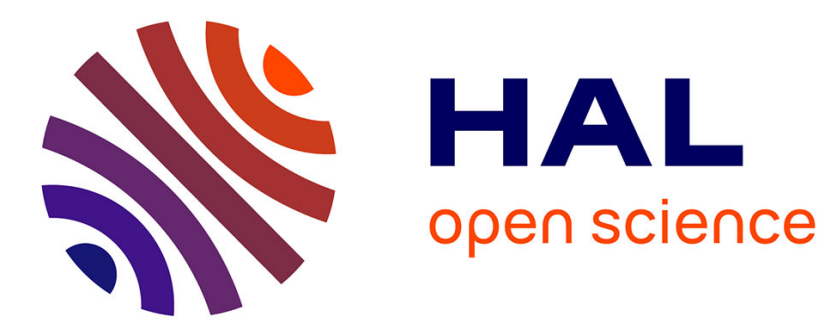

\title{
De Vargas à Collor, visages du populisme brésilien
}

Camille Goirand

\section{To cite this version:}

Camille Goirand. De Vargas à Collor, visages du populisme brésilien. Vingtième siècle. Revue d'histoire, 1997, 56 (1), pp.142-160. 10.3406/xxs.1997.4499 . hal-02560423

\section{HAL Id: hal-02560423 \\ https://hal.science/hal-02560423}

Submitted on 1 May 2020

HAL is a multi-disciplinary open access archive for the deposit and dissemination of scientific research documents, whether they are published or not. The documents may come from teaching and research institutions in France or abroad, or from public or private research centers.
L'archive ouverte pluridisciplinaire HAL, est destinée au dépôt et à la diffusion de documents scientifiques de niveau recherche, publiés ou non, émanant des établissements d'enseignement et de recherche français ou étrangers, des laboratoires publics ou privés. 


\section{De Vargas à Collor, visages du populisme brésilien}

\section{Camille Goirand}

\section{Abstract}

From Vargas to Collor: faces of Brazilian populism, Camille Goirand.

Brazil is obvioulsy a land of favor for populism. But the term there is used for many regional and national experiences. And today it's losing its force. Is it the sign of the disappearance of an exhausted political form or the announcement of its metamorphosis?

\section{Citer ce document / Cite this document :}

Goirand Camille. De Vargas à Collor, visages du populisme brésilien. In: Vingtième Siècle, revue d'histoire, $n^{\circ} 56$, octobre- $^{\circ}$ décembre 1997. Les populismes. pp. 142-160;

doi : https://doi.org/10.3406/xxs.1997.4499

https://www.persee.fr/doc/xxs_0294-1759_1997_num_56_1_4499

Fichier pdf généré le 27/03/2018 


\title{
DE VARGAS À COLLOR, VISAGES DU POPULISME BRÉSILIEN
}

\author{
Camille Goirand
}

Le Brésil est sans aucun doute une terre d'élection du populisme. Mais le terme vaut là-bas pour de nombreuses expériences régionales et nationales. Et il tend aujourd'hui à s'édulcorer. Estce le signe de la disparition d'une forme politique épuisée ou l'annonce de sa métamorphose?

$\mathrm{E}$ n mars 1974, après dix ans de dictature militaire, le général Geisel annonçait, lors de sa prise de fonction comme président de la République brésilienne, le début de la distenção, la "détente" du régime autoritaire. C'est ainsi que l'on désigne au Brésil la longue transition démocratique qui s'est achevée selon les uns en 1985 avec l'élection de José Sarney, premier président civil en vingt et un ans, selon les autres en 1989, quand Fernando Collor de Mello a été élu au suffrage universel direct; d'autres encore estiment que la démocratie brésilienne poursuit toujours en 1997 sa lente et difficile consolidation.

Alors que la libéralisation du régime commençait à devenir tangible à l'extrême fin des années 1970, deux phénomènes concomitants se sont manifestés. L'un a surpris les Brésiliens par sa nouveauté, l'autre leur a rappelé des souvenirs encore proches. À partir de 1977-
1978, l'essor des mouvements sociaux dits nouveaux a touché des domaines variés: mouvement contre la cherté de la vie, grèves des métallurgistes de São Paulo, associations de quartier ou d'aide aux enfants de rue, clubs des mères, mais aussi mouvements homosexuel ou féministe... tous sont apparus dans les grandes villes à cette époque. Dans le même temps, dès les premières élections pluralistes en 1982, des candidats populistes étaient élus aux postes laissés vacants par le régime militaire: portés aux gouvernements de Rio de Janeiro et de São Paulo, Leonel Brizola puis Jânio Quadros, anciennes figures politiques dominantes dans les années 1960, incarnaient le retour du populisme traditionnel. Fernando Collor, quant à lui, élu à la présidence en novembre 1989, en proposait une version plus modernisée.

Les manifestations historiques du populisme à travers le monde sont certes multiples et variées. Ce terme n'en désigne pas moins des phénomènes circonscrits dans le temps et l'espace, qui ont eu pour théâtre la Russie et les ÉtatsUnis du $19^{\mathrm{e}}$ siècle, ainsi que l'Amérique latine des années 1930 à 1960 ou 1970. De même que la rigueur oblige à limiter l'emploi du terme "fascisme" à l'Italie de 
Mussolini, elle incite à employer celui de "populisme" avec circonspection. Or, la chute des régimes autoritaires à l'Est comme au Sud et l'apparition de candidats à l'allure démagogue ont poussé certains analystes à proclamer le retour du populisme, sur le mode de la dénonciation. Rien ne prouve pourtant que ces "populistes" aient de réels points communs avec leurs pères et frères d'il y a quelques décennies. S'agit-il bien du même phénomène politique et social? Pour s'en assurer et pour analyser les populismes actuels, il est indispensable de faire un détour par les manifestations historiques du phénomène, autant que par sa définition.

Cet article propose un bilan de cette question pour l'Amérique latine, et en particulier pour le cas représentatif du Brésil. Y sont couramment qualifiés de "populistes", le gouvernement de Getúlio Vargas (1930 à 1937), l'Estado Novo qu'il a dirigé entre 1937 et 1945, puis sa présidence entre 1950 et 1954, celle de Jânio Quadros en 1961, ainsi que le gouvernement de Leonel Brizola à Rio de Janeiro de 1983 à $1987^{1}$. L'échec retentissant de ce dernier aux élections présidentielles d'octobre 1994 illustre par ailleurs la fin des populismes traditionnels - dont il était un représentant historique et l'émergence d'un populisme médiatique. Le nouvel aspect pris par le populisme dans les années 1980 n'est pas étranger à d'autres évolutions politiques et sociales, telles que l'émergence des mouvements populaires à partir de 19771978, la crise économique qui a frappé le pays à partir de 1981 et la mise en place des institutions de la Nouvelle République en 1988. Au moins trois évolutions se sont rencontrées dans les années 1980, avec des conséquences directes sur les formes prises par les manifestations du populisme: d'une part, l'organisation d'une partie des classes

1. Cette énumération n'est pas exhaustive. populaires au sein de mouvements actifs et autonomes, puis leur démobilisation relative à la fin des années 1980, au moment même où s'engageaient des expériences populistes; d'autre part, l'implantation d'institutions démocratiques nouvelles, vite vidées de leur sens par une classe politique qui a renoué avec ses pratiques traditionnelles, le clientélisme notamment ; l'échec, enfin, de certaines tentatives de gouvernement populiste, alors même que l'ensemble des hommes politiques avaient de plus en plus recours à des méthodes ou à un style proches du populisme. Ce sont les interactions entre tous ces facteurs, ainsi que leurs ambiguités et leurs contradictions que cet article tente de mettre en valeur, afin de répondre à la question suivante: l'émergence de larges mouvements sociaux a-t-elle constitué un facteur antipopuliste dans le Brésil de la fin des années 1980?

\section{O LE POPULISME: UN CONCEPT SPONGIEUX}

\section{Ce qu'il n'est pas}

Pour tenter de définir le populisme, on peut commencer par réfléchir sur ce qu'il n'est pas. S'il ne se fonde pas sur une idéologie cohérente, le mouvement populiste ne prend pas non plus les traits d'une organisation de classe. Les populismes, dans leurs manifestations tant russe que nord et sud-américaine, ont fait appel à des classes sociales aussi différentes que les paysans, les intellectuels, les migrants ou le prolétariat urbain. Ce simple constat suggère qu'il ne s'agit pas d'un mouvement touchant une classe sociale spécifique. Ernesto Laclau $^{2}$ relève à ce sujet que la notion de classe s'applique à un antagonisme fondé sur des relations de production: dans d'autres domaines, et en particulier en

2. Ernesto Laclau, Politics and Ideology in Marxist Theory. London, Humanities Press, 1977, 203 p. 
politique, l'antagonisme entre "classes" laisse place à une opposition entre le "peuple" et le pouvoir. Et c'est sur cette opposition aux groupes dominants que s'appuie le discours populiste. Fondé sur une double référence aux classes comme catégorie sociale et au peuple comme catégorie politique, il use d'une idéologie aux accents populaires et démocrates pour s'opposer aux groupes détenteurs du pouvoir.

Pourtant, si le discours idéologique forme un pilier du populisme, comme l'a montré Ernesto Laclau, il peine à formuler un ensemble de propositions systématiques. On peut bien sûr déceler quelques morceaux d'idéologie communs à tous les populismes. Tous proclament leur volonté de restaurer un temps situé dans un passé idéalisé, où chacun vivait sous la protection rassurante de sa communauté d'origine ${ }^{1}$, proche de ses racines. Sur ce mythe de l'âge d'or se greffe une théorie de la conspiration des élites nationales et de l'étranger contre les "petits" et les humbles, détenteurs de la vertu et de l'intégrité, qui devraient constituer le fondement de la société. Utopie conservatrice, le populisme rejette le capitalisme au nom de la défense des "gens simples", tout en exaltant à d'autres moments la nation forte et capable de s'imposer à l'extérieur grâce à un développement capitaliste impulsé par l'État. Plus qu'à un système cohérent, le discours populiste ressemble à un bric-àbrac idéologique qui s'accommode des plus folles contradictions.

Pourtant, ce fourre-tout présente une certaine unité, sans laquelle le discours populiste se verrait peut-être dépourvu de toute utilité politique. Dans le Brésil des années 1960, cet assemblage a été opéré par le nationalisme. Ainsi Fran-

\footnotetext{
1. Donald Mac Rae note judicieusement que - le populisme s'oppose à l'absence de racines. (rootlessness), dans son aricle sur "Populism as an Ideology", dans Ghita Ionescu, Ernst Gellner (eds), Populism. Its Meanings and National Characteristics, London, The Garden City Press, 1969, p. 156.
}

cisco Weffort note-t-il que le discours inaugural du très populiste Miguel Arrães, gouverneur de l'État de Pernambouco en 1961, annonce l'arrivée du "Peuple au gouvernement" et exprime la recherche angoissée d'une spécificité brésilienne et de la réalisation, à travers elle, de l'idée du peuple-communauté. Cette conception rend impensables les contradictions de classe et unit le peuple sur le fondement du sentiment d'appartenance nationale. Dans ces conditions, le nationalisme populiste sert de substitut idéologique et bloque l'émergence d'organisations de classes.

Mais surtout, l'inconsistance idéologique du populisme s'explique par un manque de place: tout l'espace est occupé par la relation directe qui se tisse entre le dirigeant et le peuple qui "S'abandonne à lui" 2 . Dans le système politique installé après la chute de Vargas en 1945, dans cette démocratie dite "de masse ", l'État, à travers ses dirigeants populistes, s'est mis en contact direct avec un peuple libéré de ses liens ruraux traditionnels. Ainsi, la pauvreté de l'idéologie populiste incite-t-elle la masse à s'en remettre à un dirigeant dont elle espère des solutions miraculeuses.

\section{Un mouvement}

Ce vide idéologique, associé à l'absence de caractère de classe du mouvement populiste, l'amène à disposer d'une large assise sociale. Plusieurs types d'interprétations, parfois complémentaires, dominent lanalyse des conditions sociales, économiques et politiques de l'apparition et de l'exercice du pouvoir par les dirigeants populistes.

Gino Germani et Torcuato Di Tella ${ }^{3}$ analysent le péronisme et le gétulisme

\footnotetext{
2. Francisco Weffort, O Populismo na Politica Brasileira, Rio de Janeiro, Paz e Terra, 1978, $181 \mathrm{p}$.

3. Gino Germani, Politica y Sociedad en una época de transición. Buenos Aires, 1965; et Torcuato Di Tella, "Populism and Reform in Latin America ", dans G. Veliz, Obstacles to Change in Latin America, London, 1970. p. 47-71.
} 
comme les effets des transitions de la société agraire vers la société urbaine industrielle, et du passage d'un système politique à représentation limitée vers une représentation élargie. Ces processus, mal synchronisés, ont provoqué des heurts car chaque transformation s'est répercutée sur les éléments encore traditionnels dans une sorte d'aller-retour entre modernisation et arriération qui a accentué chaque fois l'hétérogénéité des sociétés en transition. Selon Germani, en Amérique latine cette transition a suscité la mobilisation de groupes qui, passifs jusqu'alors, ont adopté un comportement délibératif et revendicatif nouveau, mais hors des institutions politiques et du dispositif institutionnel existants. En effet, l'entrée rapide et incontrôlée des masses marginales dans la vie politique a dépassé les capacités d'intégration offertes par le système politique et a suscité l'émergence de mouvements de mobilisation extra-institutionnels de type populiste, autour d'un chef démagogue et charismatique. Les masses rurales récemment installées en ville connaissent, ajoute Torcuato Di Tella, "une révolution d'attentes croissantes" mais insatisfaites, qui les fait tomber sous la coupe de démagogues, dont l'objectif est de contrôler et d'utiliser à leur profit la mobilisation des masses. Cette analyse du populisme, pour intéressante et répandue qu'elle soit, présente une double lacune. D'une part, elle ne se fonde sur aucune définition de la tradition et de la modernité sociales, dont la mesure est pourtant difficile. D'autre part, elle omet de se pencher sur les conditions politiques qui président à l'apparition du mouvement ou du régime populiste, ainsi que sur sa composition, pourtant très spécifique.

Octavio Ianni, pour sa part, interprète l'émergence du populisme à partir des années 1930, au Brésil, en Argentine, voire au Mexique, comme une conséquence de la crise de la domination oli- garchique ${ }^{1}$. Dans les régimes oligarchiques, les bourgeoisies liées aux productions agricoles, minières et d'élevage détenaient le contrôle du pouvoir et des institutions politiques, souvent autoritaires et répressives. Sous le choc des deux guerres mondiales et de la crise de 1929 , les oligarchies exportatrices se sont affaiblies, d'autant que les puissances étrangères sont devenues moins présentes. De leur côté, les groupes sociaux nouveaux qui émergeaient alors dans le monde urbain se sont mis à réclamer une participation politique plus ample et à remettre en question la domination des oligarchies. L'urbanisation et l'industrialisation ont ouvert dans les années 1930 des perspectives de mobilité sociale pour les classes populaires et moyennes, qui n'envisageaient donc pas la lutte en termes de classe. Dans le même temps, les bourgeoisies industrielles naissantes ont pris la direction de leurs revendications. C'est de cette alliance entre les classes populaires et la bourgeoisie nationale victorieuse des oligarchies qu'est né le populisme.

L'affaiblissement des oligarchies a donc créé un vide politique, aucune classe n'étant plus dominante, ni capable d'occuper seule le pouvoir. Le populisme est venu remplir ce vide, en se greffant sur les nouvelles conditions sociopolitiques, en forgeant une coalition temporaire, dans laquelle le dirigeant occupait une fonction d'intermédiaire et où l'iclée de "peuple" créait une fiction de solidarité. Cette nouvelle combinaison des forces au pouvoir, cette coalition hétérogène, constitue le pilier de tout régime populiste et de l'"État de compromis"2: comme aucun des groupes participant au pouvoir ne détient l'hégémonie, tous dépendent de l'État et dirigent

1. Octavio Ianni. A Formaçào do Estado Populista na América Latina. Sào Paulo. Atica, 1989. $164 \mathrm{p}$.

2. Lexpression est de Francisco Weffort, O Populismo.... op. cit. p. $56-60$. 
vers lui des attentes croissantes mais contradictoires.

Pour faire contrepoids aux grands propriétaires terriens et fonder la légitimité de son régime, Vargas s'est ainsi appuyé sur un nouvel acteur, les masses populaires. Il leur a reconnu le droit de participer, a mis en place une législation travailliste, s'est présenté comme le "père du peuple" et, enfin, a donné à l'État une fonction d'arbitre entre les différents groupes qui soutenaient le régime. Ouvert aux pressions les plus diverses, l'État populiste de masse des années 1930-1940 agissait au nom de l'intérêt national, du développement et de l'industrialisation. Autant d'objectifs formulés par une bourgeoisie qui dominait cette alliance tacite et que les masses ont soutenue tant que le régime populiste est resté légitime, c'est-à-dire tant qu'il a conservé une action d'intégration et satisfait certaines de leurs demandes.

\section{Un État}

Les régimes populistes d'Amérique latine se sont installés dans les années 1930, dans une période de mutations économiques et sociales: crise des oligarchies exportatrices, crise du capitalisme mondial, exode rural et croissance urbaine. Dans ce contexte, les dirigeants populistes au pouvoir ont engagé une profonde restructuration de l'État, de l'économie, mais aussi des relations de travail. Ils se sont attachés à promouvoir les idées de "capitalisme national" et de développement industriel, pour "rendre le pays au peuple "et lutter contre l'impérialisme. Mais cette politique aboutit surtout à un fort dirigisme, à des interventions de l'État dans l'économie et dans l'industrie notamment. S'imposent alors des politiques de substitution aux importations, fondées sur l'idée qu'une baisse des importations permettrait de diminuer la dépendance extérieure tout en stimulant la production nationale et le développement.
L'État populiste se montre donc antilibéral en économie puisqu'il monopolise la prise de décision et oriente la production industrielle. Dans les faits, le nationalisme de Getúlio Vargas ne se traduira que par la mise en ouvre de grands projets de façade destinés à donner du Brésil l'image d'une grande puissance, tels que la nationalisation des pétroles brésiliens en 1938, ou la construction des acieries de Volta Redonda à partir de 1940. C'est aussi au nom du nationalisme et du développement que Vargas engage une réforme des relations du travail, mettant en place un système "travailliste" entièrement dominé par l'État.

La mise en place d'un système corporatiste présente plusieurs avantages pour le dirigeant populiste. D'une part, elle prolonge l'emprise de l'État sur la production et le développement puisque, en intégrant les syndicats à ses organisations bureaucratiques, l'État leur ôte toute autonomie, crée un marché du travail adapté au développement des forces productives et limite les capacités revendicatives des salariés. En contrepartie, et c'est le second point, cette législation du travail donne à l'État et à son dirigeant une image de bienfaiteur du peuple. La Consolidation des lois du Travail (CLT) mise en place par Vargas en 1943 réunissait toutes les décisions travaillistes prises depuis 1930 et reconnaissait des droits sociaux importants aux salariés, tels que le salaire minimum, les congés payés, ou la protection de la maternité. Mais elle a aussi créé les conditions du pelegismo, cette symbiose des bureaucraties syndicales et de l'État, qui rend impossible l'opposition au sein des organisations de travailleurs ${ }^{1}$. En "offrant" des droits aux travailleurs, Vargas est parvenu à construire le mythe de l'État représentant du peuple et à escamoter de cette

1. Octavio Janni traite cette question dans A formaçào do Fistado Populista..., op. cit., P. 75-81 et p. 105-112. 
façon son aspect autoritaire. Surtout, il a réussi à cantonner les masses populaires dans une passivité relative puisqu'il leur était désormais inutile de revendiquer des lois sociales dont l'État prenait lui-même l'initiative.

Le populisme aboutit de cette façon à supprimer toute capacité d'action autonome des couches populaires urbaines: le dirigeant, donateur et protecteur, parle en leur nom et parvient à les manipuler, à condition de satisfaire une partie de leurs attentes. Le paternalisme qui a imprégné les relations des classes populaires et de l'État jusqu'en 1964 a fini par modeler les comportements politiques: au Brésil, toute demande de réforme est dirigée vers l'État, dans une attitude que les observateurs ont souvent jugée passive. Cependant, la frontière est ténue entre le "don" d'une loi au peuple passif et sa transformation en droit du citoyen ${ }^{1}$. Ici réside la fragilité du populisme: à partir du moment où les masses urbaines revendiquent l'application d'une loi, où elles s'affirment soit comme classe ouvrière, soit comme un ensemble de citoyens, les possibilités de manipulation diminuent et l'État populiste entre en crise, car plus rien ne pousse alors les groupes dominants à soutenir son dirigeant.

\section{Le discours d'un chef}

L'alliance tacite qui fonde le régime populiste repose sur un processus d'identification entre son dirigeant, l'État et les masses. Le chef prend figure non seulement de protecteur, mais aussi de porteparole et d'interprète du peuple. D'où l'inutilité des partis politiques, la faiblesse des organisations populistes et la dépolitisation des syndicats. Uni par un lien presque mystique avec le peuple, doté

1. À ce sujet, voir Franciscos Weffort, O Populismo.... up. cit. p. 71-76, où il analyse le rôle de la pression populaire et du passage des masses urbaines de la passivité à la revendication de droits citoyens. de vertus morales supérieures, le dirigeant charismatique incarne la souveraineté de l'État et arbitre le compromis passé entre les classes.

Pourtant, à partir du moment où les masses populaires parviennent à s'organiser hors de sa médiation, où elles donnent une forme idéologique à leurs revendications, cette identification se rompt et le dirigeant en perd le contrôle. En 1964, João Goulart n'a pas réussi à préserver son pouvoir face à la montée des organisations ouvrières qui gagnaient une autonomie croissante vis-à-vis de luimême et de l'État. La chute de "l'État de compromis" a été par ailleurs précipitée par la volte-face des groupes dominants qui, effrayés par la perspective d'une "République syndicale", ont préféré nouer une nouvelle alliance, avec les forces armées cette fois.

$\mathrm{Au}$ fondement de l'identification peuple-chef-État, le discours occupe une place primordiale dans la pratique populiste. Ernesto Laclau 2 a montré qu'il se caractérisait par l'articulation de revendications contradictoires et d'une opposition au statu quo. La notion de "peuple", bien que jamais définie, y forme le pilier du raisonnement. Ici, "peuple" désigne aussi bien la nation, le bon peuple ou "monsieur tout-le-monde", que le souverain; d'où l'utilité formidable de ce vocable qui appelle à l'unité nationale, exprime un rejet des oligarchies, des élites et des politiciens véreux, désigne le petit, souligne l'humanisme de l'homme quelconque et porte en lui de vagues promesses de gouvernement du peuple par le peuple. Le discours du "peuple" fait du dirigeant populiste le dépositaire des vertus sociales d'égalité, d'équité et de justice. Dans une communauté de sentiment, d'origine et de souffrance, le chef du mouvement populiste tente d'instaurer une relation émotionnelle entre lui

2. Frnests Laclau. Politics and Ideologyt..., op cit. 
et ses ouailles, de créer une symbiose. Le discours suscite une émotion et, par ricochets, mobilise le peuple autour de son dirigeant, dans un engagement proche de la foi.

Cette rhétorique remplit une double fonction, centrale: le peuple glorifié acquiert un sentiment d'union, d'appartenance, et une force nouvelle. Le populisme répond ici à un besoin de communauté et promeut des légitimités nouvelles. Par ailleurs, le discours se met au service du charisme du dirigeant, devenu la projection d'un idéal, entouré d'une aura et d'un mythe. Le mouvement populiste tire ainsi beaucoup plus de force du charisme de son dirigeant que d'un contenu idéologique qui se limite à l'opposition au pouvoir au nom du peuple. Et c'est grâce à cette rhétorique que le dirigeant populiste organise la mobilisation contrôlée des classes populaires, sans pour autant s'engager sur un programme gouvernemental précis.

\section{O LA RÉSURGENCE DU POPULISME}

\section{La transition démocratique au Brésil, 1974-1985}

À la mi-mars 1974 le général Geisel annonçait la détente, ou encore la décompression des institutions, contrôlée par le régime militaire. Pendant tout le processus de libéralisation par le haut ${ }^{1}$, le gouvernement a cherché à contrôler les élections et leurs résultats par une série de réformes autoritaires, tandis que les tenants de l'ouverture mettaient toute leur énergie et leur pouvoir à neutraliser la "ligne dure" des militaires, qui se serait

\footnotetext{
1. Luciano Martins, "The liberalization of Authoritarian Rule in Brazil *, dans G. O'Donnel, P. Schmitter, L. Whitehead (eds), Transitions from Authoritarian Rule, Baltimore and London, Johns Hopkins University Press, 1986, vol. 2, p. 72-94; voir aussi Thomas Skidmore, - Brazil's Slow Road to Democratization: 1974-1985", dans Alfred Stepan, Democratizing Brazil: Problems of Transition and Consolidation. New York, Oxford. Oxford Lniversity Press, 1989, p. 5-42.
}

volontiers passée de l'expérience. D'où les aller et retour constants entre libéralisation et réaction autoritaire. L'opposition conservatrice n'a été finalement muselée qu'à la fin de 1978 , après quoi la libéralisation a pu s'accélérer : le général Figueiredo a succédé à Geisel en mars 1979 et a engagé des mesures d'ouverture concrètes telles que l'amnistie des exilés politiques, le retour au multipartisme et les élections directes des gouverneurs en 1982. En 1985, les militaires ont cédé leur place à un civil à la présidence de la République et, en 1988, une nouvelle constitution a consacré le changement de régime.

L'histoire des institutions politiques brésiliennes des deux dernières décennies est celle d'une démocratisation par des moyens antidémocratiques. En effet, au cours de ces années, chaque concession du régime à l'opposition était compensée par une contre-mesure de contrôle autoritaire. Par exemple, en 1974, l'allègement de la censure et les bons résultats électoraux du Mouvement démocratique brésilien (MDB), seul parti d'opposition toléré par le régime, ont été contre-balancés par une révision du mode de scrutin pour les municipales de 1976. Les réformes avaient chaque fois pour but de bloquer l'opposition et de redonner une légitimité interne au régime, dont les dirigeants suivaient une stratégie d'ouverture limitée, alliée à une maîtrise de l'initiative politique. Les généraux ont exclu deux points de la négociation: le jugement des membres du régime et la représentation politique des groupes d'opposition radicaux. Le processus de réforme a été contrôlé par le haut car il répondait à un impératif pour les dirigeants du régime militaire : retrouver une légitimité interne et réussir à préserver, dans un contexte de crise économique grandissante, leur alliance avec les groupes dominants de l'économie. Aussi les pressions des mouvements 
populaires ${ }^{1}$ sont-elles peu entrées en compte dans la décision d'engager la détente, alors que c'est justement à ce moment-là, à la fin des années 1970, que se développaient des mouvements sociaux dirigés, d'une façon ou d'une autre, contre l'autoritarisme. En prenant eux-mêmes la décision d'abandonner le pouvoir, les militaires faisaient un double aveu d'échec : ils abandonnaient leur projet de développement, tout en reconnaissant l'inefficacité de leur politique antipopuliste. Malgré toutes les différences avec la situation d'avant 1964, les Brésiliens ont eu l'impression en 1985 de fermer la parenthèse autoritaire et d'effectuer un "retour " à la démocratie... et au populisme; sentiment renforcé par le retour du personnel politique des années 1960, jusque-là exilé.

Dans les années 1970, une grande diversité de mouvements populaires a pris son essor au Brésil et dans toute l'Amérique latine. Les associations de quartier $^{2}$, nées de comités électoraux dès les années 1950, avaient longtemps été contrôlées par le pouvoir. À la fin des années 1970, elles ont acquis une relative autonomie et formulé des revendications de plus en plus précises sur le thème de l'amélioration des conditions de vie et d'habitat, surtout à Rio de Janeiro et à São Paulo. Les Communautés ecclésiales de base (CEBs) ${ }^{3}$, elles aussi implantées dans les quartiers pauvres, ont aidé

\footnotetext{
1. Par montement poptulaire. je désigne ici lensemble des organisations daction collective de classes moyennes, ouvrières ou de facelas. Les mouvements populaires urbains se composent de citadins défavorisés, dont les revendications se centrent autour de la question des conditions de vie et des services publics : "classes populaires n est souvent ici synonyme de -peuple. de - pauvres - ou de groupes occupant une position soxiale dinfëriorité, et désigne en fait un ensemble asséz hétérogène.

2. Paul Singer, - Movimentos de Bairro-d dans P. Singer. V. (. Brant (dir.). Säo Paulo. O Poz's em .Morimentos. Petrópolis. Vozes. 1981. p. 50-70): Robert (jay. Political Clientelism and Irban Social Molements in Rio de Janeirs. P.D. Brown Iniversity. 1988, $321 \mathrm{p}$

3. Voir Warren E. Hewitt. Base Christian Communities and Secial Change in Brazil. Lincoln. I niversity of Nebraska Press. $1901.150 \mathrm{p}$
}

à la prise de conscience politique et à l'organisation de l'action collective, en particulier à partir du moment où l'Église brésilienne s'est prononcée contre le régime militaire. Enfin, le syndicalisme indépendant, apparu vers 1976, a contribué à donner une plus grande visibilité aux revendications des salariés et à les politiser. C'est pendant les grandes grèves des ouvriers métallurgistes de 1978$1980^{4}$ à São Paulo que les bases ont été jetées pour la création du Parti des Travailleurs (PT), et que Luiz Ignacio da Silva, "Lula", est devenu la grande figure politique nationale qu'il est aujourd'hui.

Pourtant, après l'effervescence des années 1970, les années 1980 ont été surtout marquées par le reflux des mouvements populaires, qui se sont progressivement intégrés au système politique ou ont été incorporés dans une lutte plus large à l'occasion de la création des partis politiques 5. Par ailleurs, l'Église brésilienne, qui a longtemps soutenu les revendications populaires, a connu un très fort virage conservateur à partir de 1978 , avec la fermeture des séminaires enseignant la théologie de la libération, la mutation des prêtres trop "rouges", l'abandon des CEBs et le retrait de la lutte politique en général. Ce déclin reste relatif car l'institutionnalisation politique des organisations populaires leur a donné des moyens de pression plus efficaces sur les administrations publiques. Si elles ne visent plus à résister au régime, elles existent encore et n'ont pas cessé de formuler de multiples revendications. Pourtant, la démocratisation n'a pas été exempte d'effets pervers pour la mobilisation: paradoxalement, la promotion

\footnotetext{
4. Voir Maria Herminia Távares de Almeida. .() sindicalismo, brasileiro entre a conservaçào e a mudancán. dans B. Sorj. M. H. T. de Almeida. Sociedade e Politica ne Brasil pos-64. Sào paulo, Brasiliense, 1983, p. 191-21.t.

5. Voir I. A. Hellman. The Study of New Social Morements and the Question of Autonomy - dans Alvare\% Escobar. The . Haking of Secial Worements in Latin America. Boulder. Wesview Press. 1992. $3 \times 3 \mathrm{p}$.
} 
de la participation des mouvements populaires au système politique et l'intégration de leurs représentants à certaines institutions, municipales en particulier, a fini par les bureaucratiser, sans pour autant favoriser partout une médiation plus efficace des demandes populaires.

Le populisme s'inscrit précisément dans ce processus et, paradoxalement, a accéléré la prise de contrôle par l'État des associations et de la mobilisation. Le cas de Rio de Janeiro permet de comprendre comment la démocratisation a pu s'accompagner de ce retour temporaire du populisme.

\section{Le "retour" du populisme dans}

les années 1980: de Brizola à Collor

\section{L'ère Chagas Freitas à Rio de Janeiro}

À partir de 1970 et jusqu'en 1982, l'État de Rio a été dirigé par l'opposition MDB de Chagas Freitas, gouverneur dit situationniste, parce qu'il entretenait de bons rapports avec les hommes au pouvoir, ou de la situation. Chagas Freitas, dont le fief électoral s'étendait sur les favelas, a mis en place dans les années 1970 un système clientéliste assez perfectionné, admirablement mis à jour par Eli Diniz 1 . Cette construction donnait au député un rôle d'intermédiaire entre la population et le pouvoir exécutif, la précarité des services donnant à l'élu des occasions répétées de transmettre personnellement les demandes de la population. Comme l'administration était débordée, seul ce jeu d'influence permettait de satisfaire les demandes de base de la population. De cette façon, le député qui parvenait à obtenir quelques améliorations bien visibles, pour un quartier précis, finissait par y acquérir une certaine légitimité. Encore aujourd'hui, les élus comme les électeurs

1. Voir Eli Diniz, Voto e Maquina Politica, patronagem e clientelismo no Rio de Janeiro, Rio de Janeiro, Paz e Terra, 1982, 228 p. En particulier, le chapitre 4, Articulando Bases de Sustentaçà) *. gardent une conception très instrumentale du vote: celui-ci ne fonde pas un système de représentation, mais constitue une monnaie dans un échange interpersonnel de faveurs ou de services.

Pour les associations des habitants des quartiers pauvres, ce système a constitué dans les années 1980 un moyen pratique pour obtenir des services auxquels elles n'auraient pas eu accès autrement. Le recours au clientélisme, ambigu, associait l'accès à une faveur, donc un rapport de dépendance personnelle, et l'utilisation stratégique et consciente de cette pratique politique par les associations, dans le seul but de voir appliquer ce qu'elles considéraient, par ailleurs, comme leurs droits. Sous Chagas Freitas, le clientélisme s'est implanté grâce au déficit persistant des services. Avec la démocratisation, les associations ont acquis une audience accrue, qui a obligé la municipalité et l'État à prendre en compte leurs revendications et donc à modifier leur mode de contrôle des organisations populaires, le simple clientélisme ayant perdu de son efficacité. À partir de 1982 et de l'élection d'un nouveau gouverneur, Leonel Brizola, la relation entre l'État et les associations de quartier s'est quelque peu modifiée. Elle illustre l'utilisation par l'État populiste de liens clientélistes, comme moyen d'asseoir son pouvoir au sein des classes populaires.

Le retour du populisme: Leonel Brizola

Ancien gouverneur de l'État du Rio Grande do Sul entre 1958 et 1964, exilé depuis lors en Uruguay puis au Portugal, président du Parti démocrate travailliste (PDT) affilié à l'Internationale socialiste depuis sa création en 1979, Leonel Brizola faisait figure, en 1982, d'homme providentiel, protecteur des petits et défenseur des libertés démocratiques. Pendant sa campagne électorale, il s'est engagé à lutter contre les inégalités, à combattre la corruption, à donner du pain aux 
enfants et à faire de l'éducation une priorité. Sa victoire à Rio de Janeiro lors des premières élections libres depuis 1965 a marqué à la fois la fin de l'ère Chagas Freitas et le début du retour à la démocratie. Elle s'explique surtout par le ton d'opposition donné à sa campagne, au cours de laquelle il déclarait "je crois dans la lutte du peuplen, à ce povo moreno (peuple brun), vite gagné par l'espoir grandissant de participer davantage à la gestion des services publiques.

Leonel Brizola a occupé le poste de gouverneur de Rio de Janeiro de mars 1983 à mars 1987, puis à nouveau de mars 1991 à avril 1994, date à laquelle il a démissionné afin de pouvoir se présenter à l'élection présidentielle. La période de son premier mandat est intéressante car, toujours dans le contexte de la démocratisation, elle coïncide avec la mise en place d'une politique sociale nouvelle, d'allure typiquement populiste, destinée aux populations les plus défavorisées, avant tout aux habitants des favelas de Rio. Au cours des opérations d' urbanisation" des favelas, entre 1983 et 1985 , le gouvernement Brizola a fait installer 223819 kilomètres de canalisations dans 74 favelas et a doté 54 favelas d'un système d'égouts. D'autres programmes ont organisé la construction de systèmes de drainage des eaux de pluie, très destructrices, ou de murs de soutènement, la mise en place de services de ramassage des ordures et l'installation d'éclairage public dans les quartiers pauvres. Enfin, il a engagé un début de légalisation des lotissements occupés par les favelados, en distribuant 3000 titres de propriété de 1983 à septembre 1985.

Outre ces travaux d'urbanisme, la politique de Brizola à Rio s'est articulée autour d'un projet central, celui des Centres intégrés d'éducation publique (CIEPs) ${ }^{1}$, éco-

1. Le gouvernement présente son programme scolaire dans Darcy Ribeiro, O Lizro Dos CIEPs. Rio de Janeiro, Bloch. 1986, 152 p. ; Escola Vica. Viz'a Fscola, revue de la commission coordinatrice de l'éducation et de la culture de l'État de Rio les destinées aux enfants des favelas et qui assurent, en plus de l'enseignement, trois repas par jour, des soins médicaux et dentaires, et une douche quotidienne. Ce sont de grandes bâtisses très imposantes, constituées de grosses masses de béton, disséminées un peu partout dans l'État de Rio, et construites par Oscar Niemeyer ${ }^{2}$. Si les CIEPs ont représenté une réponse à une situation scolaire préoccupante ${ }^{3}$, ils ont surtout été l'occasion pour Brizola de mener une politique sociale spectaculaire. Il a commencé par organiser une campagne de mobilisation autour du slogan : "Nous allons mettre l'école au net ", la rendre moins élitiste et moins injuste, en faire l'école du peuple. Il a ensuite favorisé la participation directe de la population à la rénovation des écoles, à travers la campagne "main à l'œuvre dans les écoles». À cela se sont ajoutées des distributions gratuites de lait dans les établissements scolaires et la gratuité des transports en commun pour les élèves de l'école publique. La mise en place des CIEPs a commencé dès 1984 et, en 1986, 136 fonctionnaient dans l'ensemble de l'État de Rio de Janeiro, chaque CIEP ayant une capacité de 1000 élèves.

La politique scolaire du gouvernement Brizola constitue l'exemple intéressant d'un populisme lié au clientélisme ${ }^{4}$ et

de Janeiro, 1 et 2, novembre et décembre 1983; Governo Leonel Brizola, Falas ao Professor. Ris de Janeiro, 1985. 42 p.; Governo Leonel Brizola, lim Governo que faz escola, rapport, $1986,51 \mathrm{p}$.

2. Sur les CIEPs, voir Vitor Henrique Paro (dir.), Escola de Tempo Integral, Desafio para o Ensino Público. Sào Paulo, Cortez, Autores Asscciados, 1988, 232 p.; Ana Chrystina v. Mignot, CIEPs, - Alternative para a qualidade do ensino ou nova investida do populismo na educaçào? -. thèse soutenue à l'Lniversité catholique de Rio de Janeiro, 1988. 243 p. Camille Goirand, - Des écoles pour les favelas: les CIEPs à Rio de Janeiro de 1983 à 1987 . Cabiers des Amériques Latines. 16. 1993 , p. 47-62

3. Au début des années 1980. l'échec scolaire était généralisé au Brésil, avec $60 \%$ des enfants de lécole primaire ne passant pas de la première à la deuxième année en 1984

4. Voir Vanilda Paiva. . 50 Anos do Governo Pedro Ernesto) de que espolio falamos?. Educaçào e Sociedade. 19. décembre 1984. p. 154-173 et - O Populismo e a Educaçáo no Rio de Janeiro : resposta a Darcy Ribeiro :, Educaçâs e Suciedade. 22. septembre 1986, p. 132-137. 
semble confirmer, au premier abord, l'idée d'une résurgence du populisme. Brizola s'est attaché à personnaliser l'action gouvernementale et à créer un lien direct avec ses électeurs. Par exemple, Brizolão est devenu le terme courant pour désigner les nouvelles écoles. D'autre part, les discours de Brizola et de son vice-gouverneur Darcy Ribeiro ont opéré une "génialisation" du pouvoir, propre au populisme: le "génial" Darcy a conçu les CIEPs, véritable "révolution scolaire brésilienne". Puisqu'elle est géniale, par définition, la décision du gouvernement ne se discute pas; évidence qui a favorisé un certain autoritarisme. Cette école "populaire" est offerte par un État protecteur, bienfaiteur, et dominateur: le bienfaiteur Brizola a fait cadeau des écoles à son povo moreno carioca, fournissant des "solutions" à sa grande misère.

Le populisme brizolista des années 1980 s'est appuyé sur la logique clientéliste suivante: Brizola a certes engagé des investissements sociaux d'ensemble, non sélectifs, et qui s'éloignaient en principe du système de la faveur; mais il a perpétué l'échange du vote contre des avantages matériels: chaque favela ne recevait qu'un seul programme, appliqué en deux étapes souvent séparées par une période électorale. Ainsi l'inauguration des CIEPs intervint-elle juste avant les élections de 1986: pendant la campagne électorale de Darcy Ribeiro, candidat à sa succession, Brizola a inauguré au moins un CIEP par jour, entre août et novembre. Enfin, la localisation des CIEPs dans les zones de fort regroupement d'électeurs pauvres illustre cette pratique clientéliste. Le PDT et le gouvernement de Rio ont assis leur contrôle des organisations populaires, en engageant des réformes sans consulter la population, mais en flattant ses revendications. Et c'est grâce à l'usage d'un dis- cours universel et social que le clientélisme brizolista a obtenu le soutien des associations. La politique scolaire de Brizola a donc rempli des fonctions multiples: elle a constitué une preuve de son engagement social, a favorisé une personnalisation de son pouvoir, et a renforcé son implantation électorale.

Le populisme carioca, depuis sa réapparition en 1982, a mobilisé les plus démunis en leur proposant un discours non idéologique centré sur le "peuple", avec comme contrepartie un contrôle par l'État de Rio sur les organisations populaires et donc l'affaiblissement de leurs capacités revendicatives. Ce processus s'est appuyé sur l'utilisation du budget public pour des programmes sociaux d'envergure et sur la distribution des ressources en fonction des groupes dont le pouvoir recherchait l'appui.

Dans sa forme traditionnelle, le populisme se fondait depuis les années 1930 sur l'intégration au système politique des couches les plus pauvres et les moins organisées de la population. Dans sa forme plus récente, il a aussi intégré les organisations populaires: pour ce faire, le clientélisme a permis simultanément de satisfaire leurs demandes et de faire passer la mobilisation par le prisme de l'État. L'histoire politique de l'État de Rio dans les années 1980 montre le lien qui existe entre clientélisme, mobilisation et populisme, d'une part ; entre changement de régime, retour du populisme et évolutions du clientélisme, d'autre part. Une difficulté d'interprétation subsiste cependant: même vidés de leur sens par une pratique clientéliste, les programmes sociaux menés par le gouverneur avaient une portée générale, transfigurant son style populiste en politique socialdémocrate. Du populisme subsistent chez Brizola une pratique électoraliste des programmes sociaux et surtout un discours. 
Le populisme de Fernando Collor de Mello

Fernando Collor de Mello, ancien gouverneur du petit État nordestin d'Alagoas, était presque inconnu quand il s'est lancé, au début de 1989, dans la course qui l'a mené à la présidence de la République quelques mois plus tard. La montée rapide de ce jeune candidat dans les sondages d'opinion a valu à Brizola d'être relégué à la seconde puis à la troisième place, alors qu'en 1988 il était encore donné comme possible vainqueur. Cette ascension médiatique fulgurante a poussé plus d'un observateur à qualifier Collor de populiste. Pourtant, la politique qu'il a menée pendant ses deux années au pouvoir incite à relativiser cette assertion.

Le populisme de Fernando Collor s'est construit, au cours de sa campagne présidentielle, sur une image jeune, virile et dynamique. Il répétait sa volonté de "montrer l'image d'un Brésil nouveau", d'opérer "un changement de génération"1. Jet-ski, karaté, course à pied, voyages en hélicoptère, sauts en parachute, autant de pratiques qui affichaient sa jeunesse et sa modernité. "Ne me laissez pas seul!", demandait-il à ses électeurs, leur promettant de restaurer l'union nationale et la grandeur du pays. Pour clore les discordes partisanes, Collor a prétendu incarner la nation et a refusé de participer aux querelles de ceux qui "s'occupent d'idéologie". Il s'est engagé à gouverner "pour les humbles, pour les descamisados, pour les va-nu-pieds, pour ceux qui ont été exclus du processus de développement "et a ainsi fait figure de héros salvateur, redonnant vie au messianisme de Dom Sebastião libérateur des pauvres. Il s'est ainsi présenté comme

\footnotetext{
1. Á l'occasion de recherches antéricuress, jai effectué une analyse comparative des discours et des politiques menées par les présidents Fernando Collor de Mellos au Brésil et Carlos Menem en Argentine. depuis 1989): "La résurgence du populisme dans les démocratisations d'Amérique latine ". mémosire de DEA détudes politiques, IEP de Paris. septembre 1993. $155 \mathrm{p}$. Les citations de Fernando Collor sont tiries de ce mémosire cui loon pourra en trouver les références exactes.
}

un indépendant, opposé aux "politiques" et aux élites, prêt à lutter "contre eux". Hormis ses accents justiciers, son programme de gouvernement se perdait dans le flou: "nettoyage de la machine d'État ", épuration et assainissement résument sommairement les intentions de ce caçador de Marajas, c'est-à-dire ce chasseur ou pourfendeur des parasites qui vivent de rentes publiques. La lutte contre le "corrompu", cet ennemi vague et insaisissable, focalisait les haines et les rancoeurs, et symbolisait l'opposition au "système" et aux "gros", aux élites et aux oligarchies, aux auteurs d'un imaginaire complot antipopulaire. Remarquons le caractère conventionnel et emprunté des expressions banales employées par Collor. Elles lui ont pourtant valu un grand succès auprès des foules, qui prouve son charisme, mais aussi la réussite des mises en scènes élaborées par son équipe de communication. Grâce à de savants calculs, Fernando Collor, candidat puis président, s'est doté d'un don d'ubiquité médiatique remarquable: pas un journal télévisé entre 1989 et 1992 qui ne relatait ses faits et gestes du jour. Pourtant, les thèmes de défense du peuple et de justice sociale sont restés marginaux dans le discours de ce dirigeant issu de l'oligarchie nordestine et qui a noué de contraignantes alliances électorales. À côté de ses accents populistes, à l'allure fabriquée, Collor n'a exprimé aucun désir de revanche économique sur le Nord, a rassuré plus que fustigé les créanciers internationaux du Brésil, et a annoncé sa volonté d'accélérer l'intégration du pays à l'économie internationale.

Une fois au pouvoir, le président Collor a mené une activité réformatrice fébrile, parfois précipitée et fondée sur des effets d'annonce destinés à marquer les esprits: économies budgétaires symboliques, comme la vente des voitures de fonction de la présidence, politique de choc économique, avec les plans de mai 1990 et de février 1991, ou projets de réforme 
globale de la société avec le projetão ("grand projet") de mars 1991. Fernando Collor s'est attaché, en priorité, à redéfinir le rôle de l'État en le désengageant des domaines social et économique. Son gouvernement a été marqué par de multiples réformes de structure telles que la réduction des dépenses sociales et des effectifs du personnel des administrations publiques, la suppression de la stabilité de l'emploi des fonctionnaires, les privatisations, les aides aux entreprises privées, ou l'accès de ces dernières à l'exploitation du gaz et du pétrole. La dérégulation financière s'est traduite par la mise en place de crédits à l'exportation, la baisse des taxes sur les matières premières, l'ouverture du marché intérieur aux importations et l'appel aux investissements étrangers.

Contrairement aux populistes des années 1930 à 1960, Fernando Collor s'est détourné du nationalisme économique et des politiques de relance. Ouvrant les frontières, il a mis au placard les pratiques interventionnistes de type populiste et a préféré réduire les transferts sociaux et l'engagement de l'État dans l'économie, au nom du marché. Si intention de réformes de structure il avait, elle est allée dans un sens opposé au populisme, Collor cherchant plutôt à solder l'héritage économique. Malgré sa promesse de "vaincre l'inflation par Ippon "1, et en partie à cause de l'opposition systématique du Congrès, Collor a échoué à engager le moindre redressement économique. Ainsi les opinions favorables au président ont-elles baissé de $71 \%$ en mars 1990 à $15 \%$ deux ans plus tard. Il avait alors perdu toute possibilité de se réclamer du soutien populaire ou d'une quelconque légitimité, surtout quand sa propre corruption et celle de son entourage ont été révélées en juin 1992. Formules, discours et images

1. Au karaté, passe mortelle. frappantes se sont donc avérés insuffisants à sa popularité, en l'absence de la principale ressource populiste, la légitimité du dirigeant auprès des masses populaires.

Le style politique de Collor se résume en un adage: c'est l'impression donnée qui compte, chacun pouvant en induire ce qu'il désire. Dans ce style, certains éléments présentent une forte consonance populiste: le charisme du président, ses discours enflammés, son art de la formule, sa relation apparemment privilégiée avec le peuple. Cependant, par la politique qu'il a menée, Collor s'est montré profondément non populiste, puisqu'il a accepté la dépendance économique et n'a réalisé aucun projet social d'envergure. En définitive, les accents populistes de ses discours étaient plus construits que spontanés, plus avantageux que dangereux, plus démagogues que sincères. Chez Fernando Collor, le populisme a été le résultat d'une intention stratégique publicitaire, limitée à un choix de marketing politique, loin de l'ambiguïté social-démocrate qui existe chez Leonel Brizola. Pour lui, il ne s'agissait pas tant de dire une chose et de faire son contraire que de compenser par le discours les effets négatifs sur l'électorat de décisions parfois difficiles à faire accepter, ou d'alliances avec les oligarchies tant honnies.

Au début des années 1990, ces deux épisodes politiques se sont soldés par des échecs: Fernando Collor a été l'objet d'une procédure de destitution qui l'a poussé à démissionner en décembre 1992, tandis que Leonel Brizola a perdu tout crédit tant au niveau national que carioca, après sa déroute lors de l'élection présidentielle d'octobre 1994 où il n'a pas obtenu $3 \%$ des voix. Par ailleurs, une grande proportion des hommes politiques de l'État de Rio a adopté un discours social relayé par une pratique clientéliste, avec deux conséquences: 
Brizola a perdu sa spécificité et les électeurs des classes populaires ont acquis la possibilité de faire jouer la concurrence entre les candidats.

La dictature installée en 1964 consistait certes, en partie, dans une réaction antipopuliste ${ }^{1}$. Cependant la fin véritable du cycle populiste ne se situe pas à cette date, mais plutôt vers 1985 avec l'installation des institutions démocratiques. Si Brizola a incarné les derniers sursauts d'un phénomène en voie de disparition, Collor en a proposé une pâle caricature "électronique" 2 , dans laquelle le populisme a fait place à la gesticulation médiatique et n'a pas résisté au mécontentement des électeurs. Alain Touraine notait en 1988 que la notion de "peuple" soustend la subordination de toutes les catégories sociales au projet de l'État national-populaire, ou populiste: "C'est seulement après la destruction des régimes nationaux-populaires et l'écroulement des dictatures antipopulaires que peut être entrepris le passage d'une politique de participation à une politique de représentation " 3 et que les acteurs et conflits sociaux peuvent prendre leur indépendance vis-à-vis de l'État.

Avec le retour de la démocratie, les organisations de la société civile n'ont pas toujours résisté aux tentations offertes par un clientélisme avide et encore moins aux injonctions d'un populisme

1. Octavio Ianni, dans A Formaçào..., op. cit., interprète la fin du cycle populiste comme un retournement de l'alliance tacite scellée par la bourgeoisie avec le peuple. Face à la radicalisation et à l'idéologisation des expériences populistes. la bourgeoisie appuierait la réaction autoritaire. Voir les pages 116 a 124 , intitulées - O fim do ciclo populista .

2. Lexpression est de Luiz Eduardo Soares: "Rappelonsnous que le président gouvernait sans parti, mais en s'appuyant sur les médias et sur le baromètre des sondages dopinion . Collor jouait sur une large panoplie de symboles et sur - lassociation de l'individu Fernando Collor avec des idées forces du type : énergie, initiative, audace. indépendance. persistance, réussite, agilité - dans Os Dois Coupos do Presidente. Rio de Janeiro, Relume-Dumará. 1993, p. 151-153. 3. Alain Touraine, La parole et le sang. Politique et société en Amérique latine. Paris, Odile Jacob, 1984. p. 206-207. parfois autoritaire. Pourtant, la capacité de ces dernières à résister au pouvoir impose aujourd'hui une limite sérieuse au populisme. L'émergence des mouvements populaires à partir des années 1970 et le changement de régime politique ont en effet inauguré des conditions antipopulistes nouvelles.

\section{O LA CITOYENNETÉ OCTROYÉE, HÉRITAGE POPULISTE}

\section{Le populisme édulcoré des années 1990}

Sous le coup de la démocratisation et du retour de chefs populistes dans l'arène politique, l'exercice du clientélisme a beaucoup changé. Bien sûr, dans les années 1980, le vote captif n'a pas complètement disparu, soit par fidélité soit par identification du peuple à ses vieux dirigeants comme Brizola, Quadros ou Arrães. Cependant, l'existence d'organisations communautaires fortes a imposé quelques nouveautés : d'une part, les clientèles ont changé de nature et sont désormais formées par des associations, non plus par des individus isolés; d'autre part, elles formulent de plus en plus leurs demandes en termes de droits. Enfin, les clients font aujourd'hui un usage plus conscient des réseaux de patronage et des avantages qu'ils peuvent leur apporter. Le recours au clientélisme et aux relations personnelles constitue ainsi une stratégie des associations pour obtenir ce qu'elles cherchent, autrement dit, le respect de leurs droits. Ainsi le clientélisme n'est-il pas une survivance de l'État patrimonial, ni un système en voie de disparition, héritage du passé populiste ou du monde rural. Bien plutôt, en tant que mode spécifique d'articulation entre l'État et le peuple, il est dynamique et suit le changement économique et politique: ce phénomène s'adapte, hier au contexte urbain, aujourd'hui à la 
démocratie et, tout en changeant de figure, il subsiste ${ }^{1}$.

Avec la démocratisation des années 1980 , cette adaptation s'est accompagnée de phénomènes tels que la dissociation de plusieurs niveaux politiques, un système local clientéliste coexistant avec une représentation nationale; la réapparition du populisme local comme mode de contrôle des mouvements populaires, phénomène qui rend compte en partie du reflux de la mobilisation; l'absence de véritables réformes sociales mises en place par les dirigeants des nouvelles institutions démocratiques; l'importance du discours dans la démobilisation des masses et dans leur contrôle par le chef populiste. Ce dernier point occupe une place centrale dans le populisme édulcoré des années 1990. En effet, la multiplication des promesses de réformes sociales a coupé l'herbe sous le pied aux organisations de la société civile qui les réclamaient depuis les années 1970. À Rio de Janeiro, dans les années 1980, le gouvernement Brizola a lancé de grands programmes d'urbanisation des favelas qui allaient tout à fait dans le sens des revendications déjà anciennes des associations d'habitants locales. L'expectative, mais aussi la satisfaction partielle de leurs demandes ont modéré les mouvements sociaux, qui ont alors reflué.

Ici réside la grande ambiguité et les contradictions internes du populisme des années 1990. D'une part, le dirigeant

1. Voir G. G. Bank, "Poverty, politics and the Shaping of Urban Space : a Brazilian Exemple n. International Journal of Urhan and Regional Research. 10 (4), décembre 1986, p. 523540. On peut définir le clientélisme comme un échange interpersonnel inégal, fondé sur une relation de pouvoir verticale et asymétrique, dans laquelle entre en compte un lien affectif et de kyauté entre le patron et son client. Dans les systèmes concurrentiels, les accords clientélistes s'avèrent instables et renégociables à chaque élection. À ce sujet, on pourra consulter Christopher Clapham (ed.), Private Patronage and Public Pouer: Political Clientelism in the Modern State. London. Frances Pinter, 1982, 222 p. et Jean-François Médard, "Le rapport de clientèle : du phénomène social à lanalyse politique. Retue française de science politique, 26 (1). février 1976, p. 103-131 populiste respecte les institutions démocratiques, mène une politique sociale et s'appuie sur la participation du "peuple". Cette politique a pour effet de gommer l'aspect échangiste, inégalitaire et exclusiviste du clientélisme. Dans les faits, la pratique du clientélisme ne disparaît pourtant pas car, d'autre part, l'application des programmes sociaux est soumise à des contreparties électorales et s'accompagne d'une rhétorique démobilisatrice. $\mathrm{Au}$ bout du compte, les organisations populaires y perdent toute initiative et toute autonomie. Le populisme multiplie donc les contradictions: il est à la fois social, respectueux des nouvelles institutions, mais aussi autoritaire. Ce nouveau visage témoigne de l'inachèvement et des ambiguïtés de la démocratisation au Brésil, mais aussi peut-être du caractère intrinsèquement populiste de la démocratie.

\section{Les années 1990: le style populiste}

Les aspects nouveaux empruntés par le populisme dans les années 1990 sont bien sûr liés à son adaptation aux institutions démocratiques. Mais les différences entre populismes historiques et populismes actuels sont plus profondes. D'abord, le Brésil a dépassé le stade de la transition de la société "traditionnelle" à la société "moderne", au profit d'une autre transition, celle de l'ouverture internationale. Ainsi les populistes ne mènentils plus de politiques de développement national par la substitution aux importations, mais des politiques libérales d'ajustement structurel. L'anti-impérialisme occupe aujourd'hui une fonction rhétorique et électorale, mais ne se traduit plus par des nationalisations. Au contraire, le Mexique a signé l'Alena; l'Argentine de Menem privatise ses entreprises nationales en acceptant la participation majoritaire de capitaux étrangers et le Brésil libéralise ses échanges. 
Autre différence, la coalition hétérogène entre les masses populaires et la bourgeoisie industrielle nationale, soudée par l'intermédiaire du dirigeant, n'est plus aussi évidente. Le second gouvernement Brizola (1991-1994) donne plutôt

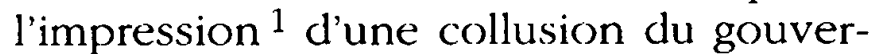
nement et des organisations illégales comme le Jogo do Bicho ${ }^{2}$ et le trafic de drogues, alors que les masses sont cantonnées à la fourniture régulière d'un vote fidèle. Les échecs ou les faiblesses du populisme brésilien des années 1990 ne s'expliquent plus par une rupture de la coalition hétérogène par une classe dominante apeurée face à la radicalisation des masses populaires, comme c'était le cas en 1964. Il s'agit davantage d'un désenchantement des classes populaires, qui, désabusées, se détournent d'un leader au charisme émoussé par l'usage répété de formules rhétoriques éculées.

Condition antipopuliste supplémentaire, la dépolitisation des masses les conduit à rejeter aujourd'hui en bloc "la politique", c'est-à-dire la corruption de la classe politique, les querelles au sein des associations de quartier et le corporatisme des syndicats traditionnels. En conséquence, le dirigeant populiste ne dispose plus des anciennes structures mobilisatrices.

Enfin, les années 1990 ne connaissent pas de crise du discours idéologique dominant, au contraire. Ernesto Laclau 3 considère en effet cette crise comme l'une des conditions d'émergence du populisme. Or, les années 1980 et 1990 ont été celles du renforcement de la démocratie et du libéralisme comme idéologies dominantes en Amérique latine et dans le monde. Le quasi-consensus démocratique qui existe entre des groupes sociaux

\footnotetext{
1. Il viagit dune impression. ou de soupcons généralisés. car aucune preure tangible n'a élé apportée jusquà présent 2. Interie clandestine

3. Erneste Laclau. Politics..., op. cit.
}

et politiques autrefois opposés a réduit le populisme des années 1980 à n'être plus que l'expression d'un sursaut de discours social des gauches, dont l'idéologie est, elle, en crise.

Dans ces conditions, depuis la démocratisation, le populisme s'est manifesté comme un simple style politique: des hommes politiques comme Brizola ou Collor l'ont utilisé comme ressource publicitaire. Dans les années 1980 et 1990, l'État populiste a perdu sa spécificité, en revanche, le style populiste s'est généralisé sous les effets de la médiatisation chu discours politique et de la massification de l'électorat. C'est ainsi que l'on a confondu l'usage d'un discours à consonances ou à interpellations populistes et un réel retour du populisme. La mise en place d'élections concurrentielles s'est en effet accompagnée d'une adaptation de l'ensemble des candidats aux méthodes modernes de marketing politique. Le populisme survit dans la mesure où il a forgé des attitudes et des comportements politiques parmi les classes populaires, où il se dilue dans les stratégies des hommes politiques et où la démocratie nouvellement installée en perpétue certains héritages. Le legs principal des régimes populistes réside dans le type de citoyenneté que connaît le Brésil et dans la permanence de la centralité de l'État dans les luttes politiques.

\section{L'béritage populiste}

Plusieurs décennies de gouvernements populistes ont laissé leur empreinte sur le système politique brésilien. Elle réside entre autres dans les particularités de la citoyenneté, étroitement liée au travail. Dans un ouvrage devenu classique, Wanderley Guilherme dos Santos a proposé le concept de "citoyenneté régulée" 4 pour montrer que, dans les années 1930,

\footnotetext{
4. Wanderley Guilherme dos Santess. Cidadania e Justica. a politica social na orde'm hrasileira. Rio de Janciro. Campus. 19:9, $138 \mathrm{p}$.
} 
la condition d'existence civique du travailleur résidait dans son appartenance à une corporation. La carteira assinada, ou carnet de travail, équivalait à un certificat de citoyenneté, octroyé par l'État et dissocié de la liberté politique. En effet, avec son emploi, le pauvre obtenait de l'État protecteur le "don" de certains droits sociaux, ce qui perpétuait la fonction tutélaire des politiques sociales ${ }^{1}$. En organisant la participation sociale et politique des classes salariées, l'État gétuliste se faisait omniprésent mais aussi ordonnateur et conducteur de la société. Dans le même temps, il enlevait aux travailleurs toute possibilité de s'affirmer en tant que sujets politiques autonomes et faisait de la citoyenneté un statut octroyé par l'État.

Cet héritage de la période populiste s'est manifesté dans le mode de transition démocratique "par le haut "que le Brésil a connu dans les années 1970 . Le changement politique a été décidé par les hautes sphères du pouvoir, qui faisaient "don" au peuple des droits démocratiques. Le changement de régime n'est pas venu à bout du clientélisme, dont la vigueur actuelle perpétue un comportement politique dépendant et une "culture politique du don"2. Les programmes sociaux sont le plus souvent perçus par leurs bénéficiaires comme un don de l'État et surtout de celui qui l'incarne. Les habitants des favelas de Rio de

1. À ce sujet, voir Vera da Silva Telles, * Pauvreté et citoyenneté, dilemme du Brésil contemporain:, Problèmes dAmérique latine, 9, avril-juin 1993, p. 73-85. Vera Telles montre comment travail et pauvreté ont fini par devenir presque synonymes, en raison des niveaux de salaire très has, ce qui rend la répartition de la citoyenneté et des droits très inégalitaire: la société brésilienne est parcosurue par une - incivilité qui s'enracine dans un imaginaire tenace sù la pauvreté est une marque d'infériorité, un mode d'être qui rend des individus incapables d'exercer leurs droits - (p. 75). Voir sa thèse, - A cidadania inexistente : incivilidade e pobreza, um estudo sobre trabalho e familia na (irande Sào Paulon, soutenue à l'Université de Sào Paulo, 1992, 335 p.

2. Cetle expression est proposée et analysée par Teresa Sales dans - Raizes da desigualdade social na cultura politica brasileira ", Revista Brasileira de Ciências Suciais, 9 (25). juin 1994 , p. 26-51.
Janeiro, par exemple, considèrent leur vote non comme un remerciement ou l'expression d'un soutien politique, mais plutôt comme une rétribution due au gouverneur qui leur a "offert " une école, attitude qui prolonge la relation d'obligation vis-à-vis du dirigeant. Le recours systématique à l'État en fait un objectif central des luttes politiques: les organisations populaires créées pour faire pression en priorité sur les pouvoirs publics n'échappent pas non plus à la pérennité des attitudes héritées de la période populiste. Pourtant, les mouvements sociaux ont aussi représenté une tentative de conquête de la citoyenneté et d'émancipation des règles imposées par les gouvernements populistes.

Malgré leur fragmentation, les organisations populaires ont réussi à jouer un rôle politique accru depuis le retrait du régime militaire. La mobilisation a poussé d'une part à la mise à l'ordre du jour des questions sociales, d'autre part à des expériences de participation populaire, tels les Conseils gouvernement-communauté proposés par Saturnino Braga, maire de Rio de 1986 à 1988. Par ailleurs, des membres d'associations d'habitants ont été élus aux Assemblées législatives des États, puis au Congrès de l'Union en 1994. C'est le cas de Chico Alencar ou de Jurema Batista, vereadores 3 entrés en politique par la mobilisation dans les associations cariocas de Sãens Peña et de la favela de Andaraí. Benedita da Silva, élue au Sénat à Brasília en octobre 1994, a elle aussi été présidente d'une association de quartier. Dans les années 1990, les revendications des mouvements populaires ont ainsi acquis une plus grande visibilité.

\footnotetext{
3. Les vereadores sont les membres des chambres municipales, qui excercent leurs compétences sur les municipios, unités administratives et politiques recouvrant un territoire bien plus vaste que nos municipalités et jouissant d'une autonomie relative depuis l'entrée en vigueur de la nouvelle Constitution fédérale, en 1988
} 
L'essor des mouvements populaires dans les années 1970 au Brésil a entraîné des changements de trois ordres: au niveau local, l'action communautaire a donné à ses participants une place dans l'espace public en tant que personnes, ce qui a pu favoriser l'émergence d'une identité sociale solidaire et citoyenne. Par ailleurs, en formulant leurs revendications en termes de droits (à l'eau, à l'électricité, à l'école...) les mouvements populaires ont contribué à la construction d'un sens de la citoyenneté ${ }^{1}$. Enfin, le système politique brésilien, traditionnellement fermé et élitiste, a engagé un semblant d'ouverture, qui s'est traduit par un discours sur la participation populaire. Même si les mouvements populaires n'ont obtenu que des résultats concrets limités, et même s'ils se sont démobilisés, ils ont contribué à la redéfinition de la lutte politique et ont mis à l'ordre du jour la question sociale ${ }^{2}$. Ils ont ainsi participé à l'érosion de l'élitisme traditionnel du système politique brésilien et de l'héritage populiste, à la consolidation de la jeune démocratie et à la construction d'une citoyenneté nouvelle.

\section{L'impossible populisme des années 1990}

Par leur existence même, les mouvements populaires ont marqué le système politique brésilien et en ont changé certaines règles du jeu. Dans les années

1. Je développe ce thème dans un article intitulé - Citoyenneté et pauvreté dans le Brésil de la Nouvelle République ", à paraitre dans un prochain numéro de Latin American Perspectives.

2. Scott Mainwaring, - Urban Popular Movements, Identity and democratization in Brazil . Comparative Political Studies, 20(2), juillet 1987, p. 131-159. Il écrit à ce propos: " Même si les mouvements ne peuvent se targuer que de victoires limitées, ils nen redéfinissent pas moins les paramètres de la lutte politique... Tel ou tel mouvement peut bien disparaître ou se démobiliser, il produit tout de même d'importants changements dans les relations sociales et dans le système politique * (p. 153). *Les conquêtes matérielles de ces mouvements, quoique importantes pour les participants, ont été limitées et partielles. Néanmoins, les mouvements populaires urbains ont contribué à redéfinir la scène politique de multiples manières. notamment en augmentant la sensibilité du public à légard des revendications populaires. (p. 154).
1990 , ils ont constitué le principal facteur antipopuliste car leur visibilité et leur autonomie ont rendu leur contrôle bien plus ardu pour les dirigeants populistes. L'autonomie, réelle ou mythique, des organisations de la société civile a détaché le "peuple" de son chef. En effet, l'impulsion donnée par ce dernier s'est montrée moins nécessaire à la mobilisation que dans les années 1930 ou 1950. Dans les années 1930, le dirigeant populiste instrumentalisait le peuple pour satisfaire des revendications que les classes moyennes avaient exprimées dans les années 1920; dans les années 1980, les classes populaires ont formulé de façon autonome leurs propres revendications. La conclusion tacite d'une alliance pluriclassiste autour d'un dirigeant populiste en a donc été compliquée. Le mythe de l'autonomie des mouvements populaires souligne leur volonté ou même leur pouvoir d'échapper au contrôle d'un dirigeant extérieur à eux-mêmes, et donc de créer des conditions antipopulistes. Par l'action collective, les classes populaires ont pris des initiatives, se sont affirmées, ont participé, ont innové et se sont imposées comme sujet collectif ayant des droits. En conséquence, la tutelle de l'État, cet héritage des régimes populistes, s'est affaiblie et les mouvements populaires ont constitué un élément à la fois de construction citoyenne et de dépassement du populisme.

L'ensemble de ces conditions explique pourquoi le phénomène populiste a perdu sa force pour se transformer en un néopopulisme médiatique, qui n'a de points communs avec ses ancêtres des années 1930 que le discours et un effort d'instrumentalisation des couches populaires. Aujourd'hui, la légitimité des dirigeants tient bien davantage à la nature démocratique du régime politique qu'à l'identification des politiciens à un peuple mythifié. Sous les effets conjugués des mouvements populaires, de la démocra- 
tisation, de la contrainte idéologique néolibérale et de la médiatisation, le populisme a été réduit à l'état de ressource électorale utile à la démagogie de candidats en quête de suffrages, mais qui ne ressemble que de très loin, et par la forme seulement, à ses antécédents.

En s'appuyant sur les médias, les populistes se sont imposé leur propre limite puisqu'ils se sont exposés à la surveillance des citoyens-téléspectateurs. Dans un cercle vicieux, la médiatisation de la politique suscite, nourrit, mais aussi limite le populisme: à travers le petit écran, le dirigeant sollicite le contrôle des citoyens, s'auto-contrôle, modère donc sa politique, mais dans le même temps l'omniprésence des médias constitue la principale ressource populiste. En conséquence, les pratiques populistes se répandent au sein de la classe politique, mais elles sont sans substance, diluées, édulcorées. S'opposant à la classe dirigeante traditionnelle mais respectant les nouvelles institutions, acceptant les contraintes de la crise mondiale et se pliant aux mécanismes de la démocratie, jouant de la personnalisation et de la médiatisation de la politique, les nouveaux dirigeants populistes du Brésil ont inauguré dans les années 1980 une variante lointaine de ce phénomène, compatible avec la compétition et l'incertitude démocratiques.

Aujourd'hui, nombre d'hommes politiques se voient qualifiés de "populistes", terme qui, dans le vocabulaire politique actuel, marque d'indignité et d'irrespectabilité ceux qui en sont affublés. Pourtant, le phénomène est souvent l'objet d'une lecture trop rapide, qui le confond avec un amalgame de démagogie et de médiatisation. Qualités devenues indispensables à tous les hommes politiques, l'usage expert des médias et l'excellence oratoire ne suffisent pas au populisme, nous l'avons vu. La maîtrise des techniques modernes de communication, avatar lointain du style populiste, s'impose aux hommes politiques et les transforme tous un peu en descendants des caudillos des années 1930 à 1960. La plupart d'entre eux restent étrangers, pourtant, au populisme tel qu'il s'est alors manifesté en Amérique latine.

Allocataire de recherche au CERI de la Fondation nationale dessciencespolitiques, enseignanteàl'Institut d'études politiques de Paris, Camille Goirand achève sa thèse consacrée aux mouvements populaires et à la démocratisation à Rio de Janeiro de 1970 à nos jours. 